\title{
Value of 4D MR Angiography at 3T Compared with DSA for the Follow-Up of Treated Brain Arteriovenous Malformation
}

\author{
S. Soize, F. Bouquigny, K. Kadziolka, C. Portefaix, and L. Pierot
}

\begin{abstract}
BACKGROUND AND PURPOSE: Four-dimensional, contrast-enhanced MRA is a useful technique for the diagnosis and classification of brain AVM. The purpose of this study was to evaluate its usefulness in the follow-up of treated brain AVM.
\end{abstract}

MATERIALS AND METHODS: Patients with treated brain AVM (embolization, radiosurgery, and/or surgery) were investigated with both DSA (the "gold standard") and 4D MRA. Four-dimensional MRA was performed at 3T using a 4D sequence, combining contrast-enhanced timing-robust angiography, keyhole, and sensitivity encoding techniques. Examinations were evaluated by 2 independent readers and disagreements were resolved by a third reader. Interobserver and intermodality agreement with respect to residual nidus, residual venous drainage, and brain AVM patency were determined.

RESULTS: Between May 2008 and February 2013, 37 patients with a median age of 45 years (interquartile range $=26-55$ ) were prospectively included. Examinations were acquired 36 months (IQR $=10-45.5)$ after the last treatment. Interobserver agreement for brain AVM patency was very good for both 4D MRA $(\kappa 0.82,95 \% \mathrm{Cl} .67-.98)$ and DSA $(\kappa 0.84,95 \% \mathrm{Cl} .69-.98)$. After consensus reading, intermodality agreement for the evaluation of brain AVM patency was good $(\kappa 0.73,95 \% \mathrm{Cl} .55-.90)$. Diagnostic accuracy of 4D MRA for residual brain AVM compared with DSA, reached a sensitivity of $73.7 \%$, specificity $100 \%$, positive predictive value $100 \%$, and negative predictive value $78.3 \%$. Agreements by technique of treatment are also detailed.

CONCLUSIONS: Four-dimensional MRA is a useful radiation-free technique for the follow-up of patients with treated brain AVM, especially patients treated by radiosurgery. However, given its actual limitations it is not sufficient to assert the cure; DSA remains mandatory for this purpose.

ABBREVIATIONS: 4D-MRA $=4 \mathrm{D}$ contrast-enhanced MRA; bAVM = brain AVM; CENTRA = contrast-enhanced timing-robust angiography; SENSE $=$ sensitivity encoding

B rain AVMs (bAVMs) are rare developmental disorders (prevalence of $0.01 \%-0.52 \%$ ) composed of a network of channels (nidus) interposed between feeding arteries and draining veins. ${ }^{1}$ They are often revealed by spontaneous intracranial hemorrhages with high morbidity and mortality. ${ }^{2,3}$ They are often diagnosed in young, otherwise healthy patients and carry a life-long bleeding risk ranging between $1.5 \%$ and $4.0 \%$ per year. ${ }^{4,5}$ There are several treatment options, including surgical resection, endovascular embolization, and radiosurgery, that can be attempted to achieve a cure. $^{6-10}$ The risk of bleeding persists as long as an anatomic cure is not completely achieved. ${ }^{5,6}$ Therefore, it is necessary to confirm

Received January 3, 2014; accepted after revision February 27.

From the Department of Radiology, Hôpital Maison Blanche, Université de Champagne-Ardenne, Reims, France.

Please address correspondence to Laurent Pierot, MD, PhD, Department of Radiology, Maison Blanche Hospital, 45 Rue Cognacq-Jay, 51092 Reims, France; e-mail: Ipierot@gmail.com

http://dx.doi.org/10.3174/ajnr.A3982 an AVM cure after the treatment. When the AVM is treated by surgery and/or embolization, imaging is performed a few weeks or months after the end of the treatment to evaluate the AVM occlusion status (complete occlusion or residual AVM). When AVM is treated with radiosurgery (alone or after embolization and/or surgery), occlusion is usually achieved after a few years, and a regular imaging follow-up is conducted every 6 or 12 months.,

Despite several disadvantages, such as radiation exposure, iodinated contrast agent injection risks (including allergy and nephrotoxicity), and neurologic procedural risks $(0.30 \%-$ $2.63 \%),{ }^{11-13}$ DSA is the reference method to evaluate AVM occlusion status because of posttreatment modifications. Indeed, it has a very good spatial and especially temporal resolution allowing for a precise evaluation of potential residual nidus and residual venous drainage. To reduce these risks, several noninvasive crosssectional imaging techniques like 3D TOF-MRA and 3D contrastenhanced MRA were used. ${ }^{14-16}$ The diagnostic accuracy of these techniques was quite good, but not enough to replace DSA be- 
cause they suffered from a poor temporal resolution that limited analysis of the angioarchitecture and hemodynamics. ${ }^{14-16}$ Fourdimensional contrast-enhanced MRA (4D-MRA) was conceived to solve this problem and to provide a better temporal resolution while also preserving spatial resolution. However, the first examinations were not efficient enough; by incorporating progressive new technologies such as parallel imaging and intelligent sampling of the $k$-space, it became an emergent imaging technique with the advantage of a dynamic "DSA-like" evaluation of the AVM that allows for the definition of arterial feeders, nidus, and draining veins in the same examination. ${ }^{17}$ Actually, 4D-MRA permits the effective diagnosis and classification of bAVM. ${ }^{17-20}$ The value of 4D-MRA for the follow-up of patients with a treated bAVM has been rarely evaluated. ${ }^{21,22}$ The purpose of this study was to evaluate the performance of $4 \mathrm{D}-\mathrm{MRA}$ at $3 \mathrm{~T}$ for the follow-up of patients with a treated bAVM. In other words, this study will try to answer the 2 following questions: is 4D-MRA enough to assert the cure? Is 4D-MRA useful for the follow-up of treated bAVM?

\section{MATERIALS AND METHODS Study Design}

Institutional review board approval was obtained and informed consent was waived according to the design of the study. All patients treated for a bAVM in our academic department were prospectively included in a data base. Since May 2008, all patients receiving MR imaging in our department for a bAVM underwent a 4D-MRA. For the purpose of the present study, patients imaged between May 2008 and February 2013 were included if they met the following criteria: 1 ) they had a bAVM treated at least by 1 of the following modalities (embolization, radiosurgery, or surgery), 2) they were imaged during the follow-up with both 4D-MRA at $3 \mathrm{~T}$ and DSA, 3) both examinations were performed at least 1 month after the last treatment, 4) both examinations were performed in a time interval less than 1 month and without treatment in between, and 5) image datasets were of sufficient quality. For patients who underwent several sets of examinations, only the last set was taken into account to avoid redundant data.

\section{Treatment Strategy}

For all patients analyzed in this study, indication and strategy of treatment was based on multidisciplinary decisions involving neurologists, neurosurgeons, radiotherapists, and neuroradiologists.

\section{MR Imaging}

Examinations were performed on a 3T whole-body MR imaging system (Achieva; Philips Health Care, Best, the Netherlands), using an 8-channel sensitivity encoding (SENSE) head coil that allows for parallel imaging. A dose of $0.2 \mathrm{~mL} / \mathrm{kg}$ of gadoterate meglumine (Dotarem; Guerbet, Aulnay-sous-Bois, France) was injected at a flow rate of $1.5 \mathrm{~mL} / \mathrm{s}$ using an automated power injector (Spectris; Medrad, Indianola, Pennsylvania), followed by a saline flush of $30 \mathrm{~mL}$ injected at the same flow rate $(1.5 \mathrm{~mL} / \mathrm{s})$.

The 4D-MRA was performed with 20 dynamic sagittal acquisitions using a 3D, T1-weighted, fast-field echo sequence. Imaging parameters were as follows: TE, $1.50 \mathrm{~ms}$; TR, $2.9 \mathrm{~ms}$; flip angle, $20^{\circ}$; section thickness, $1 \mathrm{~mm}$; acquisition bandwidth, $1350 \mathrm{~Hz}$;
FOV: $270 \times 126 \times 150 \mathrm{~mm}^{3}$, covering all of the brain; acquisition matrix, $264 \times 200 \mathrm{~mm}^{2}$; and reconstruction matrix, $400 \mathrm{~mm}$. A total of 160 thin sagittal $2-\mathrm{mm}$ sections with a $1-\mathrm{mm}$ overlap between sections were acquired with an acceleration SENSE factor of 2 in the selection direction and a SENSE factor of 2.7 in the phase-encoding direction, yielding a total acceleration factor of $5.4(2.7 \times 2) .{ }^{23}$ Half-Fourier imaging was used, skipping $25 \%$ of $k$-space data, yielding an acceleration factor of 1.33. For further acceleration, we used combined contrast-enhanced timing-robust angiography (CENTRA)-keyhole $k$-space filling with a percentage factor of $18 \%$ and the last dynamic scan as reference, yielding an acceleration factor of 5.55. ${ }^{17,24}$ Combining these techniques, the 4D-MRA yielded a total acceleration factor of 40 [5.4 (SENSE) $\times$ 5.55 (18\% keyhole) $\times 1.33$ (half scan factor)] compared with standard contrast enhanced-MR angiography without such techniques. In total, 20 dynamic acquisitions were achieved with a temporal resolution of 1.4 seconds per volume, combined with a reference scan of 7.8 seconds, for a total acquisition time of 34.8 seconds, with a spatial resolution at acquisition of $1.02 \times 1.13 \times$ $2 \mathrm{~mm}^{3}$ and after interpolation a voxel size of $0.68 \times 0.68 \times 1$ $\mathrm{mm}^{3}$.

\section{Conventional Angiography}

DSA was performed on a biplane angiography unit (Axiom Artis; Siemens, Erlangen, Germany) with a 5-Fr catheter using a femoral artery approach and selective contrast injection of the internal carotid artery, external carotid, and vertebral arteries. A temporal resolution of 3 images per second was used, in standard orthogonal anteroposterior and lateral projections and oblique projections, if necessary. Anteroposterior images had a matrix of $720 \times$ $720 \mathrm{~mm}^{2}$ and FOV of $32 \mathrm{~cm}$. Lateral projections had a matrix of $960 \times 960 \mathrm{~mm}^{2}$ and FOV of $25 \mathrm{~cm}$. Three-dimensional rotational angiography was not systematically performed and these data were not used in this study. Bolus injections of iodinated contrast material (iodixanol, Visipaque 320; GE Healthcare, Vélizy-Villacoublay, France) were $6 \mathrm{~mL}$ at a flow rate of $2 \mathrm{~mL} / \mathrm{s}$ for the external carotid, $8 \mathrm{~mL}$ at a flow rate of $4 \mathrm{~mL} / \mathrm{s}$ for the internal carotid, and $10 \mathrm{~mL}$ at a flow rate of $5 \mathrm{~mL} / \mathrm{s}$ for the vertebral artery using an automated power injector (Angiomat Illumena; Liebel-Flarsheim Medical System, Cincinnati, Ohio).

\section{Image Analysis}

A first reader (4 years of experience in diagnostic and interventional neuroradiology) evaluated the 4D-MRA overall image quality before image analysis using a practical 5-point grading system formerly used by Hadizadeh et al. ${ }^{19}$ Examinations were considered of sufficient quality if the score was $\geq 3$. Examinations with a score lower than 3 were excluded.

Two independent readers ( 11 and $>20$ years of experience in diagnostic and interventional and neuroradiology, respectively) reviewed 4D-MRA and DSA examinations randomly. Readers were blinded to baseline and follow-up clinical data and were only aware of the bAVM location and the received treatments. They determined if there was a residual nidus and/or residual venous drainage for each examination. Results for each reader were recorded separately and used to determine interobserver and intermodality agreement. For studies where the 2 readers disagreed, 


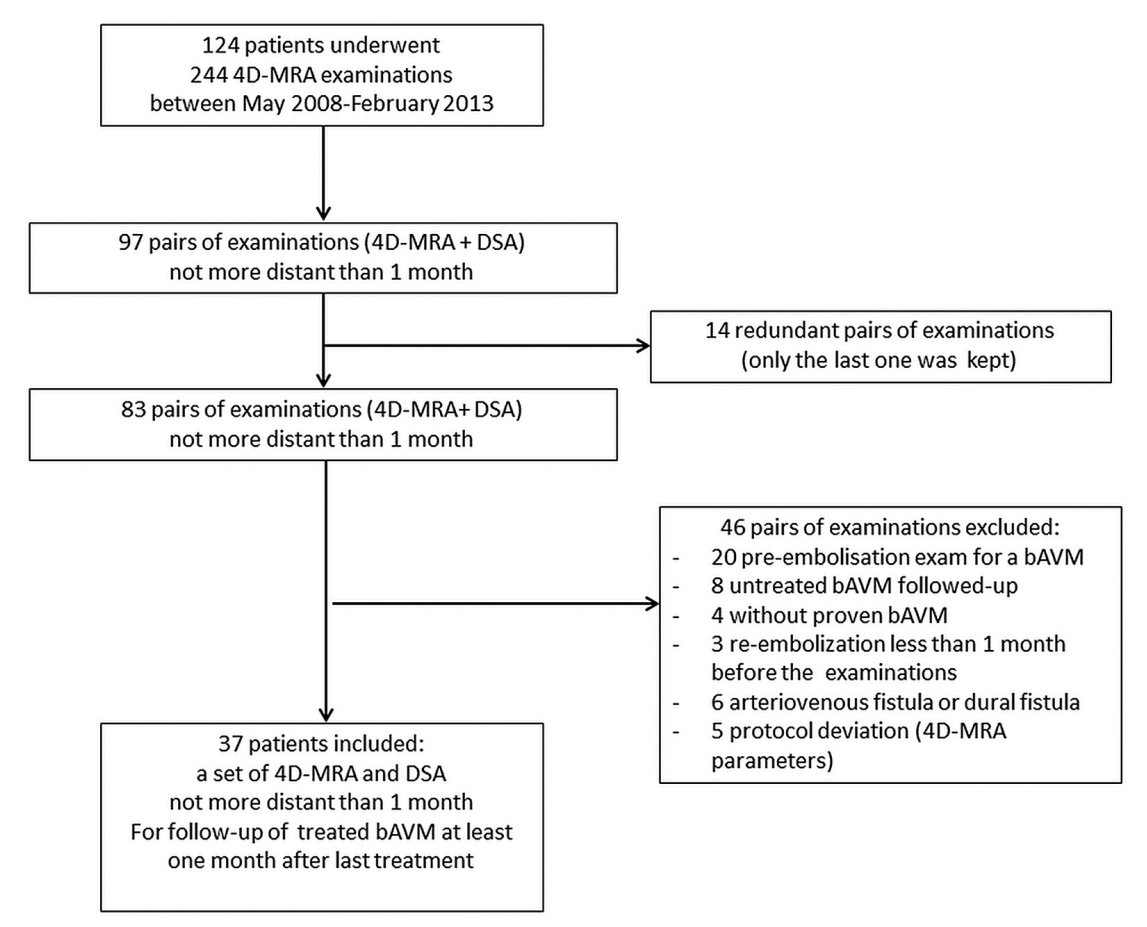

FIG 1. Patient flow chart. DF indicates dural fistula.

Table 1: Characteristics of the 37 patients

\begin{tabular}{lc}
\hline \multicolumn{1}{c}{ Demographics } & \\
\hline Age, years & $45.0[26.0-55.0]$ \\
Sex, male & $22(59.5 \%)$ \\
Type of treatment & \\
Embolization then radiosurgery & $14(37.8 \%)$ \\
Embolization & $8(21.6 \%)$ \\
Radiosurgery & $8(21.6 \%)$ \\
Surgery & $3(8.1 \%)$ \\
Surgery then radiosurgery & $3(8.1 \%)$ \\
Surgery then embolization & $1(2.7 \%)$ \\
bAMV location & \\
Supratentorial & $33(89.2 \%)$ \\
Infratentorial & $4(10.8 \%)$ \\
Time intervals & \\
Delay between last treatment and & $36[10-45.5]$ \\
images, months & \\
Delay between DSA and 4D-MRA, days & $1[0.5-1]$ \\
Presentation & \\
Hemorrhage & $16(45.7 \%)$ \\
Seizure & $8(22.9 \%)$ \\
Headache & $6(17.1 \%)$ \\
Neurologic symptoms & $4(11.4 \%)$ \\
Incidental & $1(2.9 \%)$ \\
\hline
\end{tabular}

Note:-Continuous variables were presented by proportion and percentage, categoric variables were described with median, first, and third quartiles. Presentation mode was available for 35 of 37 patients.

consensus for each imaging technique was performed by a third reader $(>20$ years of experience in diagnostic and interventional neuroradiology), who was blinded from the results of the first readings and used to determine intermodality agreement. A residual bAVM was defined as the presence of a residual venous drainage associated or not with a residual nidus.

All MRA and DSA data were transferred to the local PACS and were randomly reviewed on separate workstations using the medical viewer. For 4D-MRA visualization, subtracted data with MIP reconstructions were used. The software enabled the enlargement of regions of interest in any $3 \mathrm{D}$ direction, with the possibility of viewing the progress of the contrast injection. For DSA visualization, each stored run was composed of a loop of 6 images, which enabled the readers to appreciate the contrast filling with discrimination of arterial, capillary, and venous phases. Enlargement of the regions of interest was permitted by the software as for MRA images.

\section{Statistical Analysis}

Quantitative variables were described as mean and standard deviation, whereas qualitative variables were described as numbers and percentages. Interobserver agreement for 4D-MRA and DSA and intermodality agreement with respect to the presence of residual nidus, residual early venous drainage, and bAVM patency were determined using the $\kappa$ statistic test. Results were displayed as percentages and $95 \%$ CI. Strength of agreement was defined as $\kappa \leq 0.20$ : poor; $\kappa$ 0.21-0.40: fair; $\kappa$ $0.41-0.60$ : moderate; $\kappa 0.61-0.80$ : good; $\kappa 0.81-0.90$ : very good; and $\kappa \geq 0.91$ : excellent, including 95\% CI. In addition, the exact number and percentage of results from the 2 readers and the 2 modalities that were in exact agreement were provided. A $P$ value of less than .05 was considered statistically significant. Sensitivity, specificity, and positive and negative predictive values were then calculated. All statistical analyses were performed with MedCalc statistical software (11.4.3.0; MedCalc Software, Mariakerke, Belgium).

\section{RESULTS}

Between May 2008 and February 2013, 37 patients fulfilled the inclusion criteria (Fig 1). Their demographic data, different types of treatment, bAVM location, time intervals (between the last treatment and images, and between both imaging modalities), and presentation mode are detailed in Table 1. For patients who underwent radiosurgery, mean time between images and irradiation was $3.7 \pm 2.0$ years (extremes: $24-144$ months).

\section{Image Quality}

Image quality scores ranged between 3 (adequate for diagnosis) and 5 (excellent), with a mean quality score of $3.6 \pm 0.5$ on the 5 -point grading scale. All examinations were judged to be of adequate quality for diagnosis.

\section{Interobserver Agreement}

For 4D-MRA examinations, the 2 observers were in agreement for residual nidus in 35 of 37 (94.6\%) bAVMs, resulting in good interobserver agreement $(\kappa 0.78,95 \%$ CI .57-.98). The 2 disagreements concerned small $(<1 \mathrm{~cm})$ and heterogeneous residual nidus in patients treated by embolization. The readers matched for 

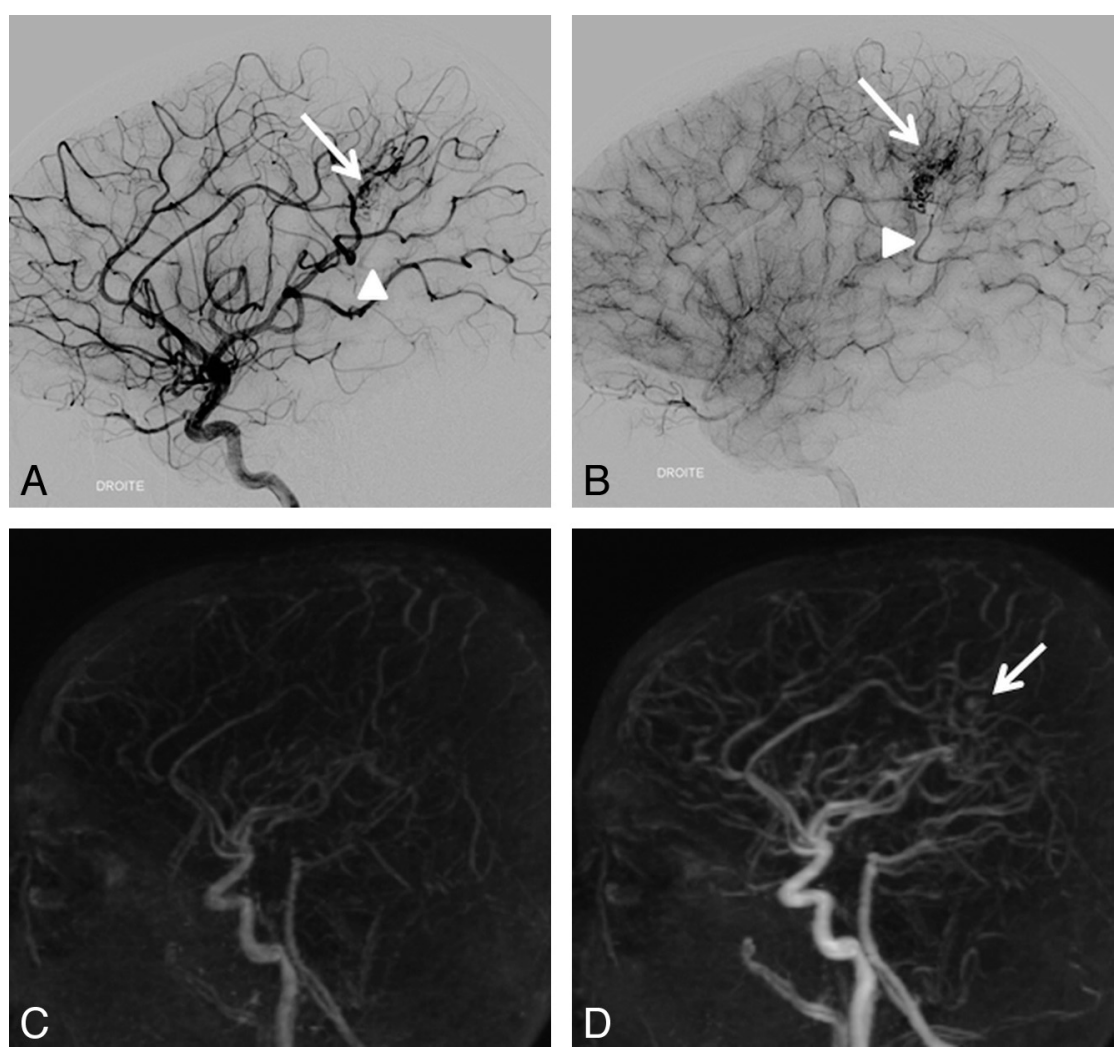

FIG 2. An 11-year-old boy with a right pericallosal AVM revealed by hemorrhage and treated 11 months ago by surgery. Residual nidus (arrow) and venous drainage (arrowhead) are seen on DSA $(A, B)$ but not on 4D-MRA $(C, D)$ by both readers. Retrospectively, a part of the nidus may be visible (arrow).
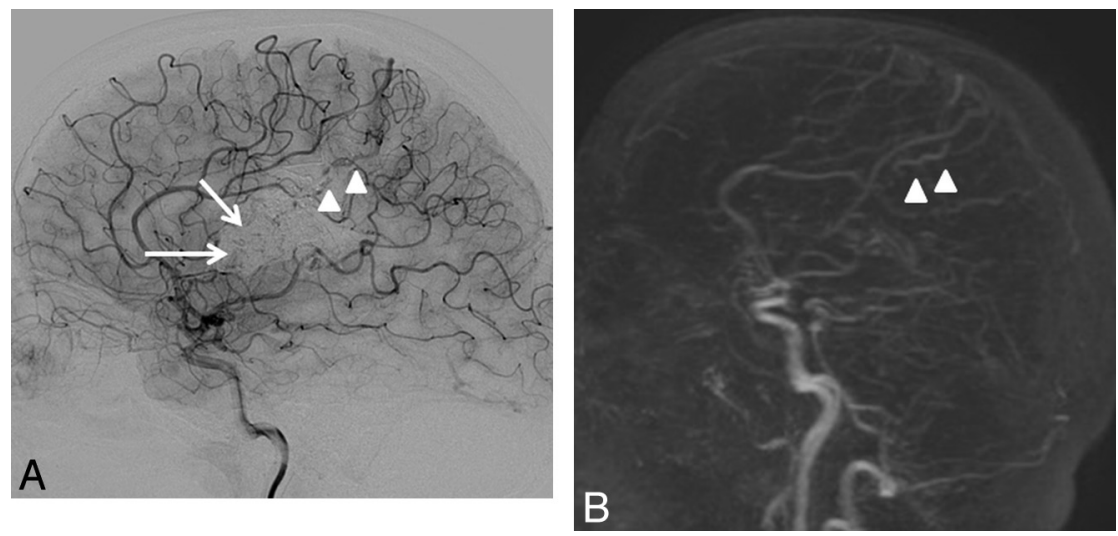

FIG 3. A 32-year-old woman with a left frontal-parietal AVM revealed by seizures and treated by embolization then radiosurgery more than 4 years ago. Residual nidus (arrow) and venous drainage (arrowhead) are seen on DSA ( $A$ ) but not on 4D-MRA (B) by both readers. Retrospectively, the draining vein is visible (arrowhead) on 4D-MRA, but is not dilated and appears during the venous phase (indistinguishable from cortical veins).

residual venous drainage in 34 of 37 (91.9\%) bAVMs, also resulting in good interobserver agreement ( $\kappa$ 0.68, 95\% CI .44-.91). The 3 disagreements concerned small veins, of which 1 was visible early and 2 were visible only since the capillary phase. When taking into account only the patency of the bAVM (residue or cured), agreement was very good ( $\kappa 0.82,95 \%$ CI .67-.98).

For DSA examinations, observers were in agreement for residual nidus in 35 of 37 (94.6\%) bAVMs, yielding good interobserver agreement ( $\kappa 0.80,95 \%$ CI .61-.99). Two patients had a very small residual nidus masked by a large cast of Onyx not seen by 1 of the readers. The observers matched in 34 of 37 (91.9\%) bAVMs for residual venous drainage, also resulting in good interobserver agreement $(\kappa 0.68$, 95\% CI .48-.92). Disagreements concerned 3 patients with veins that were visible during the capillary phase and interpreted as normal by 1 reader. Taking into account only AVM patency (residue or cured), agreement was very good $(\kappa 0.84$, 95\% CI .69-.98).

\section{Intermodality Agreement}

Consensus readings for 4D-MRA and DSA showed agreement regarding residual nidi in 33 of 37 (89.2\%) bAVMs, resulting in moderate intermodality agreement ( $\kappa 0.60,95 \%$ CI .35-.85). The unseen nidi were small (4 of 4, 100\%) and heterogeneous ( 3 of $4,75 \%$ ), which is often seen when the AVM has been embolized (4 of 4, 100\%). Figures 2 and 3 show examples of nidi and veins undiagnosed with 4D-MRA. For residual venous drainage, consensus readings showed agreement in 32 of 37 (86.48\%) bAVMs, also resulting in moderate agreement ( $\kappa$ 0.51, 95\% CI .25-.78). Concerning the unseen venous drainage, 4D-MRA had a worse accuracy when veins were not dilated (3 of 5, $60 \%)$ and seen only during the capillary phase on DSA (3 of 5, 60\%). In addition, unseen residual venous drainage on MRA was always masked by a large cast of Onyx that was not completely transparent and diminished the visibility of pathologic veins. In contrast, when the drainage was early, the vein dilated, and the nidus compact, depiction was easier (Fig 4). When taking into account only the patency of the bAVM, the agreement was good ( $\kappa$ 0.73, 95\% CI .55-.92).

\section{Diagnostic Value of 4D-MRA Compared with DSA}

The diagnostic accuracy of 4D-MRA reached a sensitivity of $73.7 \%$, specificity $100 \%$, positive predictive value $100 \%$, and negative predictive value $78.3 \%$.

\section{Agreement for Patients Treated by Radiosurgery or Embolization}

Among patients treated by radiosurgery alone (8/37, 21.7\%), the diagnostic accuracy reached a sensitivity of $100 \%$, specificity $100 \%$, positive predictive value $100 \%$, and negative predictive value $100 \%$. Among patients treated by embolization alone $(8 / 37$, 

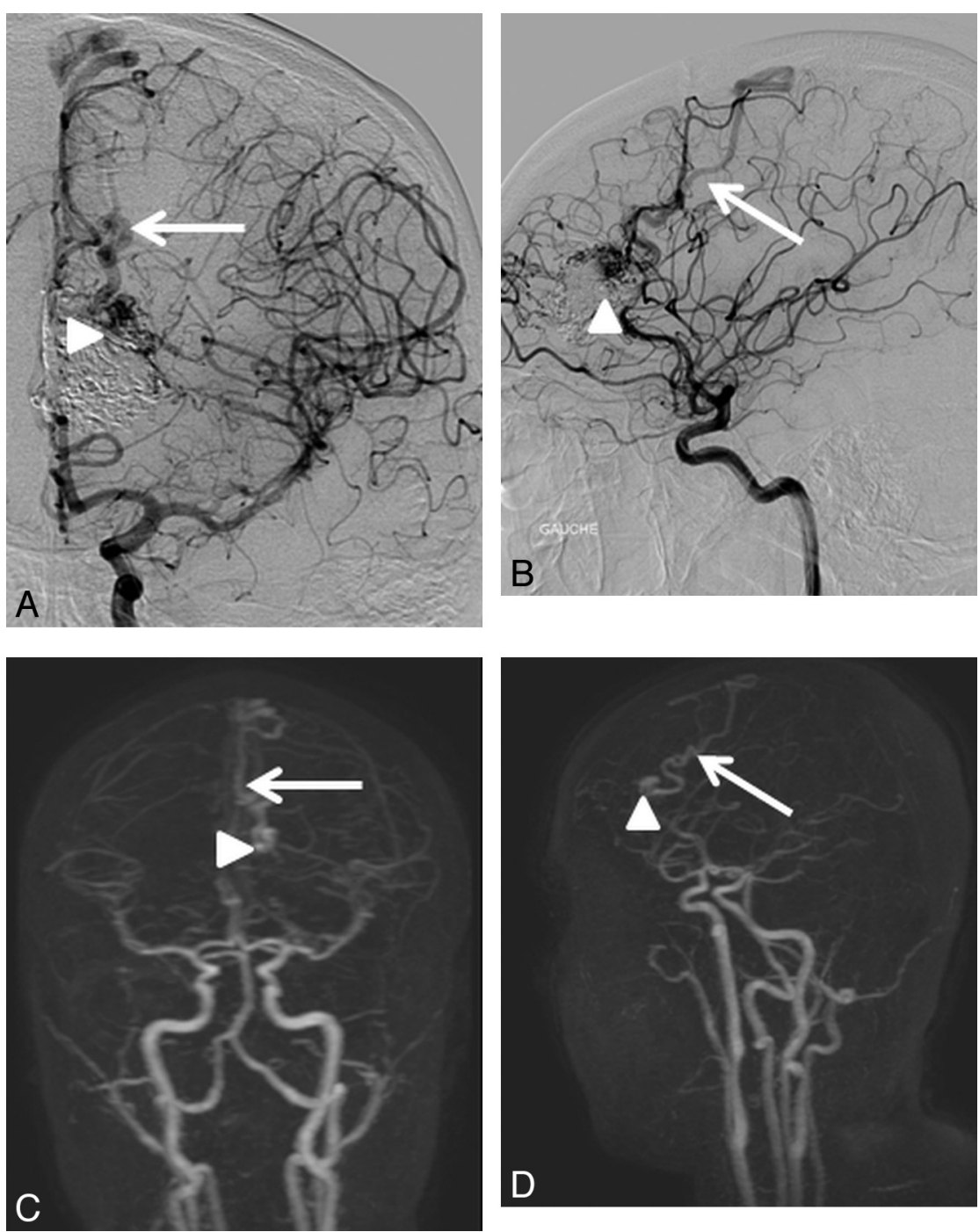

FIG 4. A 16-year-old adolescent girl with a left frontal AVM revealed by transitory neurologic symptoms; treated by embolization then radiosurgery 2 years and 10 months earlier. When the drainage is early, the vein dilated, and the nidus compact, depiction is easy. The residual nidus (arrowhead) and venous drainage (arrow) were visualized by both readers in DSA $(A, B)$ and 4D-MRA $(C, D)$.

\section{Table 2: Agreement for patients treated by radiosurgery or embolization}

\begin{tabular}{lll}
\multicolumn{1}{c}{ Parameters by Modality of Treatment } & \multicolumn{1}{c}{$\begin{array}{c}\text { Residual } \\
\text { Nidus }\end{array}$} & $\begin{array}{c}\text { Residual Venous } \\
\text { Drainage }\end{array}$ \\
\hline $\begin{array}{l}\text { Radiosurgery alone }(n=8) \\
\text { Interobserver agreement for 4D-MRA }\end{array}$ & $1.00(1.00-1.00)$ & $1.00(1.00-1.00)$ \\
$\quad$ Interobserver agreement for DSA & $1.00(1.00-1.00)$ & $1.00(1.00-1.00)$ \\
$\quad$ Intermodality agreement between 4D-MRA and DSA & $1.00(1.00-1.00)$ & $1.00(1.00-1.00)$ \\
$\begin{array}{l}\text { Embolization then radiosurgery ( } n=14) \\
\quad \text { Interobserver agreement for 4D-MRA }\end{array}$ & $0.75(0.43-1.00)$ & $0.75(0.43-1.00)$ \\
$\quad$ Interobserver agreement for DSA & $0.50(0.08-0.92)$ & $0.75(0.43-1.00)$ \\
$\quad$ Intermodality agreement between 4D-MRA and DSA & $0.49(0.06-0.92)$ & $0.50(0.08-0.92)$ \\
Embolization alone $(n=8)$ & $0.50(0.00-1.00)$ & $1.00(1.00-1.00)$ \\
$\quad$ Interobserver agreement for 4D-MRA & $0.50(0.00-1.00)$ & $0.20(0.00-0.81)$ \\
Interobserver agreement for DSA & & \\
Intermodality agreement between 4D-MRA and DSA & $0.50(0.00-1.00)$ & $0.50(0.00-1.00)$ \\
\hline
\end{tabular}

Note:-Data are given as percent $(95 \% \mathrm{Cl})$.

$21.7 \%$ ), the diagnostic accuracy reached a sensitivity of $75.0 \%$, specificity $100 \%$, positive predictive value $100 \%$, and negative predictive value $80.0 \%$. Furthermore, for patients treated by embolization combined with radiosurgery $(14 / 37,37.8 \%)$, diagnos- tic accuracy reached a sensitivity of $66.7 \%$, specificity $100 \%$, positive predictive value $100 \%$, and negative predictive value $62.5 \%$. Interobserver and intermodality agreements for these 3 groups of patients are detailed in Table 2.

\section{Patient Outcomes}

On follow-up DSA, 18 of 37 (48.6\%) patients had a residual AVM and the remaining 19 patients had no residual AVM. In the group treated by embolization, 8 of $22(36.4 \%)$ were cured. Eight patients $(21.7 \%)$ who were treated by embolization and complementary radiosurgery had small residuals followed-up (pending the effectiveness of radiosurgery); 2 patients $(5.4 \%)$ had a new embolization attempt; 3 patients (8.1\%) had additional radiosurgery; 1 patient $(2.7 \%)$ had a second session of complementary radiosurgery; and 3 patients $(8.1 \%)$ refused further treatment. In the group treated by radiosurgery alone, 6 of 8 (75.0\%) were cured. Moreover, in the surgery group, 5 of 7 (71.4\%) were cured.

\section{DISCUSSION}

Four-dimensional contrast-enhanced MRA is increasingly used in bAVM assessment as it shows high efficacy to make a diagnosis and anatomic analysis of bAVM, with good interobserver and intermodality agreements compared with DSA. It is actually an emerging noninvasive imaging technique to assess bAVM and can potentially help to reduce the number of postinterventional invasive diagnostic DSAs. However, its role in posttreatment follow-up is actually not well-defined. The present study evaluated the accuracy of 4D-MRA at 3T for detecting residual bAVM after treatment. The results showed that $4 \mathrm{D}-\mathrm{MRA}$ had good interobserver agreement $(\kappa 0.78$, 95\% CI .57-.98 for residual nidus and $\kappa$ $0.68,95 \%$ CI $.44-.91$ for residual venous drainage), moderate intermodality agreement with DSA $(\kappa 0.60,95 \%$ CI $.35-.85$ for residual nidus and $\kappa 0.51,95 \%$ CI .25.78 for residual venous drainage), and good overall agreement $(\kappa 0.73,95 \% \mathrm{CI}$ $.55-.92$ ), yielding to a sensitivity of $73.7 \%$, specificity $100 \%$, positive predictive value $100 \%$, and negative predictive value $78.3 \%$.

Although it has good diagnostic accuracy, given its actual limitations, a negative 4D-MRA cannot assert the AVM is cured with 
certainty. Subgroup analysis showed that the diagnostic accuracy seemed to be better in patients treated by radiosurgery alone than those who underwent embolization (alone or in combination with radiosurgery). The excellent agreement ( $\kappa 1.00,95 \% \mathrm{CI}$ 1.00-1.00) for patients treated after radiosurgery could compete with DSA for the follow-up of these patients. These results are consistent with the study of Lim et $\mathrm{al}^{22}$ which focused on the diagnostic accuracy of 4D-MRA after radiosurgery and found a diagnostic accuracy of $86.1 \%$, with a sensitivity of $79.6 \%$ and $64.3 \%$ (reader 1 and 2), a specificity of $90.9 \%$ and $100 \%$, a positive predictive value of $84.6 \%$ and $100 \%$, and a negative predictive value of $90 \%$ and $81.5 \%$. Furthermore, even if 4D-MRA cannot depict all the residual bAVM, its very high sensitivity and positive predictive value make it a very reliable test in case of positivity. This means that if it detects a residual bAVM, there is no risk of a false-positive and it can potentially help avoid some unnecessary DSA examinations, especially for patients followed-up after radiosurgery (see agreements for patients treated by radiosurgery or embolization).

Hadizadeh et $\mathrm{al}^{21}$ recently concluded an excellent correlation between 4D-MRA and DSA regarding pre- and postoperative evaluation of bAVM, suggesting that $4 \mathrm{D}-\mathrm{MRA}$ could be substituted for DSA in these 2 indications. However, in this series, of the 25 patients who had been followed-up after treatment, only 1 patient presented residual venous drainage. In our study, the proportion of residual bAVM detected with DSA was higher; 18 of 37 (48.6\%), of which 12 of 18 (66.7\%) were correctly depicted by 4D-MRA. Our results are consistent with the study of Lim et al, ${ }^{22}$ which focused on the follow-up after radiosurgery. However, this study, compared with the present study, had the limitation of a large delay (up to 6 months) between the realization of 4D-MRA and DSA images. In contrast to the absence of false-positives in our study, Lim et $\mathrm{al}^{22}$ reported 1 in their series where a normal vein was interpreted as pathologic. Indeed, sometimes it is difficult to differentiate if a vein is normal or pathologic. To avoid subjective disagreements on the physiopathology of pathologic veins, in our study, veins were considered pathologic by both readers when they appeared early (ie, during the arterial or capillary phase). Despite this, during the capillary phase, there are many superimpositions that can lead readers to miss some pathologic veins because of the lack of temporal and spatial resolution. This is particularly true for 4D-MRA but can also be seen in some cases with DSA.

The interobserver agreement on DSA was very good but a bit less than at 4D-MRA because of difficult cases (very small residual AVM) in which 4D-MRA was negative for both readers, whereas the residual AVM was sometimes seen by 1 reader on DSA leading to more disagreement. The third reader who made consensus determined that there was no false-positive case and disagreements were based on missed veins and/or nidus that were particularly difficult to depict. This problem was particularly true for patients treated by embolization with a large cast of Onyx and a small residual AVM. Indeed, there were 2 disagreements on DSA related to 2 small residual AVMs that were very difficult to identify (they were not seen on 4D-MRA by both readers) and missed by 1 reader on DSA; these 2 difficult cases did not cause disagreement on 4D-MRA but were responsible of disagreement for DSA. The results of the subgroup analysis should be interpreted with caution for this reason and because the number of patients was small in each group so that any disagreement may strongly vary the result.

Four-dimesional contrast-enhanced MRA showed moderate agreement with DSA regarding the depiction of residual nidus and venous drainage. However, unseen residues were either small and heterogeneous nidus or veins appearing on capillary time. In our study, residual bAVMs unseen with 4D-MRA were difficult to diagnose and concerned mainly patients who were treated by embolization followed by gamma knife. Five patients benefited from a new treatment after the results of 1 set of examinations: 2 embolization attempts, 2 additional treatments by gamma knife in patients previously treated by embolization, and 1 patient treated with a second session of radiosurgery. There is, therefore, a diagnostic and therapeutic impact in properly monitoring these patients.

After radiosurgery, obliteration of bAVM is progressive and achieved after 2 to 5 years. ${ }^{25}$ Given that these patients need repeated examinations during a long-term follow-up, an annual 4D-MRA can be proposed until it becomes negative (ie, until there is no residual AVM visible). Then a final DSA examination can be done to assert the cure. In contrast, if 4D-MRA shows a residual bAVM after a long follow-up (maybe 4 or 5 years, the delay in discussing the failure of radiosurgery treatment is still controversial), a DSA examination will be needed to decide on future therapeutic options (new radiosurgery or other treatments can be considered). ${ }^{26}$ For follow-up after radiosurgery, this scheme accords with the conclusion of Khandanpour et $\mathrm{al}^{27}$ (except that they used TOF-MRA instead of 4D-MRA). After surgery, the bAVM is most often cured, but sometimes removal is incomplete. In these cases, DSA seems to be the appropriate method to assert the cure or to plan new treatments (in case of incomplete surgery). After embolization, given its actual limitations and strengths, 4D-MRA has a limited place. When the bAVM is not cured after the last embolization, there is a need to do a DSA for evaluation before future treatment. When the bAVM seems to be cured at the end of the last embolization session, it can be useful to use 4D-MRA for the follow-up. If it is positive (ie, residual bAVM is visible), then DSA will be performed with a new attempt at embolization, and, if it is negative, a late DSA will be performed to assert the cure.

Recently, several publications described new tools that can further improve the efficacy of 4D-MRA, opening new prospects for bAVM assessment before treatment. Four-dimensional flow MRA techniques provide velocity measures and even color-encoded flow maps to aid in understanding the angioarchitecture. $^{28,29}$ In addition, the contribution of selective arterial spinlabeling, in addition to 4D-MRA, provided supplementary functional or anatomic information in $25 \%$ of the cases. ${ }^{30}$ These techniques have not been evaluated in the posttreatment population.

It is also important to specify the limits of our study. First, the same technique developed in 2008 was used during the study period. However, although this technique is actually currently used and accords to recent publications, there is still the possibility of further improving the spatiotemporal resolution of this technique. Indeed, a way of improving our sequence could be to per- 
form hemi-encephalic imaging, with the advantage of a better temporal resolution while minimizing vessel superimpositions. In addition, temporal and spatial resolution can be further improved up to an acceleration factor of 60 by optimizing the use of intelligent $k$-space sampling techniques (ie, CENTRA, parallel imaging, and partial Fourier imaging), whereas our examinations had an acceleration factor of 40 , equivalent to other recent publications. ${ }^{17,19-21,28,31}$ Second, the interobserver agreement for DSA was not as high as expected compared with 4D-MRA, though it was very good $(\kappa 0.84,95 \%$ CI $.69-.98$ and $\kappa 0.82,95 \%$ CI .67-.98, respectively) for the reasons explained above.

\section{CONCLUSIONS}

Four-dimensional contrast-enhanced MRA is a useful noninvasive technique for the follow-up of treated bAVM, especially in patients treated by radiosurgery. However, given its actual limitations it is not sufficient to assert the cure. DSA remains mandatory for this purpose.

Disclosures: Laurent Pierot has provided consultancy for Codman, Covidien/ev3, MicroVention, Penumbra, and Sequent.

\section{REFERENCES}

1. Cognard C, Spelle L, Pierot L. Pial arteriovenous malformations. In: Forsting M, ed. Intracranial Malformations and Aneurysms. Berlin/ New York: Springer-Verlag; 2004:39-100

2. Choi JH, Mohr JP. Brain arteriovenous malformations in adults. Lancet Neurol 2005;4:299-308

3. Da Costa L, Wallace MC, Ter Brugge KG, et al. The natural history and predictive features of hemorrhage from brain arteriovenous malformations. Stroke 2009;40:100-05

4. Kondziolka D, McLaughlin MR, Kestle JR. Simple risk predictions for arteriovenous malformation hemorrhage. Neurosurgery 1995; 37:851-55

5. Pierot L, Cognard C, Spelle L. Cerebral arteriovenous malformations: evaluation of the hemorrhagic risk and its morbidity. J Neuroradiol 2004;31:369-75

6. Novakovic RL, Lazzaro MA, Castonguay AC, et al. The diagnosis and management of brain arteriovenous malformations. Neurol Clin 2013;31:749-63

7. Pierot L, Januel AC, Herbreteau D, et al. Endovascular treatment of brain arteriovenous malformations using Onyx: preliminary results of a prospective, multicenter study. Interventional Neuroradiology 2005;11(suppl 1):159-64

8. Pierot L, Januel AC, Herbreteau D, et al. Endovascular treatment of brain arteriovenous malformations using Onyx: results of a prospective, multicenter study. J Neuroradiol 2009;36:147-52

9. Pierot L, Cognard C, Herbreteau, et al. Endovascular treatment of brain arteriovenous malformations using a liquid embolic agent: results of a prospective, multicentre study (BRAVO). Eur Radiol 2013;23:2838-45

10. Pierot L, Kadziolka K, Litré F, et al. Combined treatment of brain AVMs with use of Onyx embolization followed by radiosurgery. AJNR Am J Neuroradiol 2013;34:1395-400

11. Kaufmann TJ, Huston J, III, Mandrekar JN, et al. Complications of diagnostic cerebral angiography: evaluation of 19,826 consecutive patients. Radiology 2007;243:812-19

12. Fifi JT, Meyers PM, Lavine SD, et al. Complications of modern diagnostic cerebral angiography in an academic medical center. $J$ Vasc Interv Radiol 2009;20:442-47

13. Thiex R, Norbash AM, Frerichs KU. The safety of dedicated-team catheter-based diagnostic cerebral angiography in the era of ad- vanced noninvasive imaging. AJNR Am J Neuroradiol 2010;31: 230-34

14. Heidenreich JO, Schilling AM, Unterharnscheidt F, et al. Assessment of 3D-TOF-MRA at 3.0 Tesla in the characterization of the angioarchitecture of cerebral arteriovenous malformations: a preliminary study. Acta Radiol 2007;48:678-86

15. Unlu E, Temizoz O, Albayram S, et al. Contrast-enhanced MR 3D angiography in the assessment of brain AVMs. Eur J Radiol 2006;60: 367-78

16. Lee KE, Choi CG, Choi JW, et al. Detection of residual brain arteriovenous malformations after radiosurgery: diagnostic accuracy of contrast-enhanced three-dimensional time of flight MR angiography at 3.0 Tesla. Korean J Radiol 2009;10:333-39

17. Taschner CA, Gieseke J, Le Thuc V, et al. Intracranial arteriovenous malformation: time-resolved contrast-enhanced MR angiography with combination of parallel imaging, keyhole acquisition, and k-space sampling techniques at 1.5 T. Radiology 2008;246:871-79

18. Parmar H, Ivancevic MK, Dudek N, et al. Neuroradiologic applications of dynamic MR angiography at 3 T. Magn Reson Imaging Clin N Am 2009;17:63-75

19. Hadizadeh DR, von Falkenhausen M, Gieseke J, et al. Cerebral arteriovenous malformation: Spetzler-Martin classification at subsecond-temporal-resolution four-dimensional MR angiography compared with that at DSA. Radiology 2008;246:205-13

20. Machet A, Portefaix C, Kadziolka K, et al. Brain arteriovenous malformation diagnosis: value of time-resolved contrast-enhanced MR angiography at 3.0 T compared to DSA. Neuroradiology 2012;54: 1099-108

21. Hadizadeh DR, Kukuk GM, Steck DT, et al. Noninvasive evaluation of cerebral arteriovenous malformations by 4D-MRA for preoperative planning and postoperative follow-up in 56 patients: comparison with DSA and intraoperative findings. AJNR Am J Neuroradiol 2012;33:1095-101

22. Lim HK, Choi CG, Kim SM, et al. Detection of residual brain arteriovenous malformations after radiosurgery: diagnostic accuracy of contrast-enhanced four-dimensional MR angiography at 3.0 T. Br J Radiol 2012;85:1064-69

23. Weiger M, Pruessmann KP, Kassner A, et al. Contrast-enhanced 3D MRA using SENSE. J Magn Reson Imaging 2000;12:671-77

24. Willinek WA, Gieseke J, Conrad R, et al. Randomly segmented central k-space ordering in high-spatial-resolution contrast-enhanced MR angiography of the supraaortic arteries: initial experience. $R a$ diology 2002;225:583-88

25. Steiner L, Lindquist C, Adler JR, et al. Clinical outcome of radiosurgery for cerebral arteriovenous malformations. J Neurosurg 1992;77:1-8

26. Maesawa S, Flickinger JC, Kondziolka D, et al. Repeated radiosurgery for incompletely obliterated arteriovenous malformations. J Neurosurg 2000;92:961-70

27. Khandanpour N, Griffiths P, Warren D, et al. Prospective comparison of late 3T MRI with conventional angiography in evaluating the patency of cerebral arteriovenous malformations treated with stereotactic radiosurgery. Neuroradiology 2013;55:683-87

28. Ansari SA, Schnell S, Carroll T, et al. Intracranial 4D flow MRI: toward individualized assessment of arteriovenous malformation hemodynamics and treatment-induced changes. AJNR Am J Neuroradiol 2013;34:1922-28

29. Illies T, Forkert ND, Ries T, et al. Classification of cerebral arteriovenous malformations and intranidal flow patterns by color-encoded 4D-hybrid-MRA. AJNR Am J Neuroradiol 2013;34:46-53

30. Kukuk GM, Hadizadeh DR, Boström A, et al. Cerebral arteriovenous malformations at $3.0 \mathrm{~T}$ : intraindividual comparative study of $4 \mathrm{D}$ MRA in combination with selective arterial spin labeling and digital subtraction angiography. Invest Radiol 2010;45:126-32

31. Willinek WA, Hadizadeh DR, von Falkenhausen M, et al. 4D timeresolved MR angiography with keyhole (4D-TRAK): more than 60 times accelerated MRA using a combination of CENTRA, keyhole, and SENSE at 3.0 T. J Magn Reson Imaging 2008;27:1455-60 